\title{
THE USE OF ENDOSCOPIC DIAGNOSIS IN DOGS WITH UPPER RESPIRATORY DISEASES WITH RESPECT TO THE LOCALISATION OF PATHOGENS AND THE SUBSEQUENT THERAPY
}

\author{
Bajtoš, M., Kožár, M. \\ University of Veterinary Medicine and Pharmacy in Košice, Komenského 73, 041 81, Košice \\ Slovakia \\ martin.kozar@uvlf.sk
}

\section{ABSTRACT}

Bacterial diseases of the upper respiratory tract accompanied with various degrees of clinical signs are relatively frequent in a small animal clinical practice. The clinical signs are usually mild, mostly connected with clinical manifestation of nasal discharge, mild dyspnoea, sneezing, and coughing; however, in some cases they may convert to a chronic stage with serious systemic manifestations. The course and development of complications depends on the etiological agent and the success or failure of the subsequent therapy. An accurate diagnosis is of the upmost importance in order to develop an appropriate therapeutic plan for disease management. The present study focused on: endoscopic visualisation of the upper respiratory tract of the affected animals; localisation of pathological changes, and the targeted collection of the samples. This clinical study involved 26 patients with long-term signs of the affected airways that progressed to chronic stages after the failure of the prescribed therapy. Each patient was clinically examined, sedated and subjected to an endoscopic ex- amination that involved the collection of samples. The samples were examined microbiologically, tested for antibiotic resistance, and a targeted therapy was implemented. The endoscopic and complex microbiological diagnosis enabled: more effective management of the bacterial infection; shortening of the therapy; and a convalescence period that reduced the risk of the development, or the spreading of resistant bacteria.

Key words: bacterial infection; laryngotracheitis; rhinitis; samples cultivation and endoscopy visualisation; upper respiratory diseases

\section{INTRODUCTION}

Infectious diseases of the upper canine respiratory tract constitute a significant worldwide health issue encountered in a veterinary clinical practice. The affected group includes senior dogs and also young dogs with reduced immunity, development disorders and chronic degenerative changes. Both acute and chronic infections of the up- 
per respiratory airways frequently lead to a wide range of clinical signs. The most frequent are: mucopurulent nasal discharge, sniffling, as well as coughing associated with the excitation of the patient and dyspnoea that can also be related to diseases of other organ systems [9].

The etiology of the development of respiratory diseases is frequently associated with various causative agents, such as bacteria, viruses, fungi, parasites and other pathogens. The respiratory tract is constantly exposed to these infectious agents either by aerogenic or haematogenic pathways. The pathogenic invasion is arrested by physical, chemical and immunological mechanisms that include mucin, mucocilliary clearance and various inherent antimicrobial factors [15]. The impairment of the protective mechanisms caused, for example, by immunosuppression, stress, toxins, environment and infectious agents, frequently results in the development of chronic inflammatory processes affecting individual compartments of the respiratory tract.

In the majority of cases, rhinitis as a disease of the upper respiratory tract is frequently overlooked during the primary diagnosis. From the etiological point of view, rhinitis is most frequently induced by the propagation of viral infections. However, secondary bacterial contaminations may occur in long term inflammation or chronic diseases. In such conditions connected with untreated cases, inflammation from rhinitis may spread to the surrounding areas. The subsequent progressive chronic rhinitis involves infiltration of the inflammatory process into the surrounding structures and the development of sinusitis maxillaris, frontalis and infraorbitalis [11]. A non-specific infectious rhinitis is very rare in dogs. Other factors participating in the development of inflammation of the upper respiratory tract include nasal trauma, allergy, or the penetration of a foreign object [15].

Laryngotracheitis (Fig. 1) is another disease characterised by the inflammation of the upper respiratory tract. The etiology of this disease is polyfactorial. The pathogens that most frequently cause this disease include: bacteria (Bordetella bronchiseptica, Mycoplasma cynos, and Streptococcus equi subsp. zooepidemicus), and viruses (canine parainfluenza virus CAV-2, canine adenovirus CAV-1, canine influenza virus, and canine respiratory virus). The primary way of pathogen transfer involves aerosol and the clinical signs appear about 3-10 days after the exposure to the pathogens. Additional sources of infection are: inadequate sanitation of surfaces, contaminated examination instruments, endotracheal tubes, and toys [3].

The correct diagnosis plays an important role in the complex of prognosis, selection of therapy and its effectiveness. Endoscopic imaging diagnosis is a targeted diagnostic method. The advantages of this method are: the visualisation of the internal structures of the nasal and frontal cavities, larynx, pharynx, trachea and bronchi, the identification of anatomic abnormalities, and also the lo-

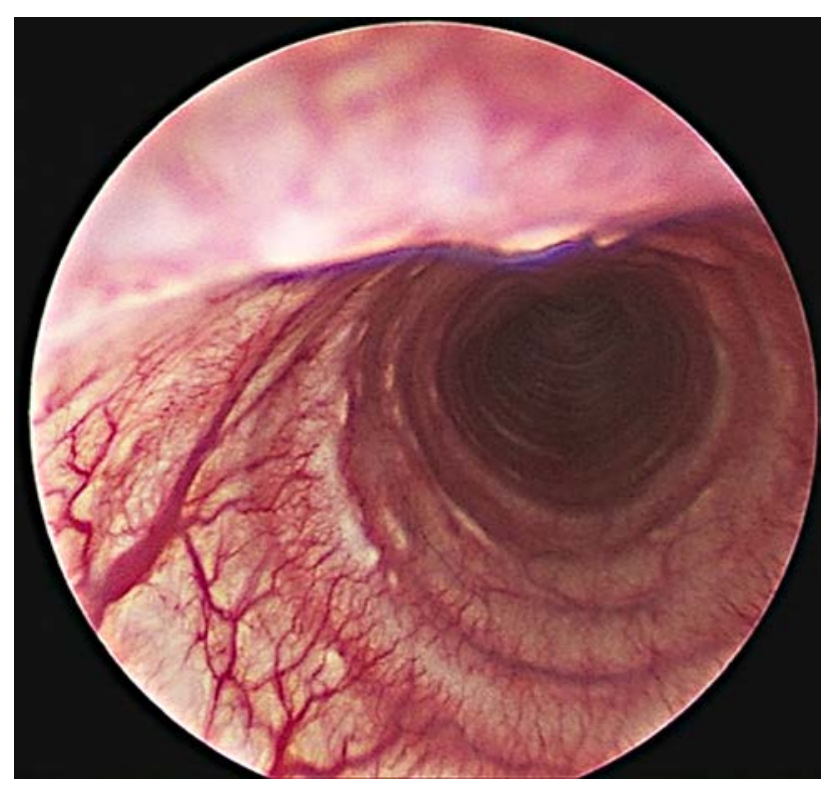

Fig. 1. Laryngotracheitis

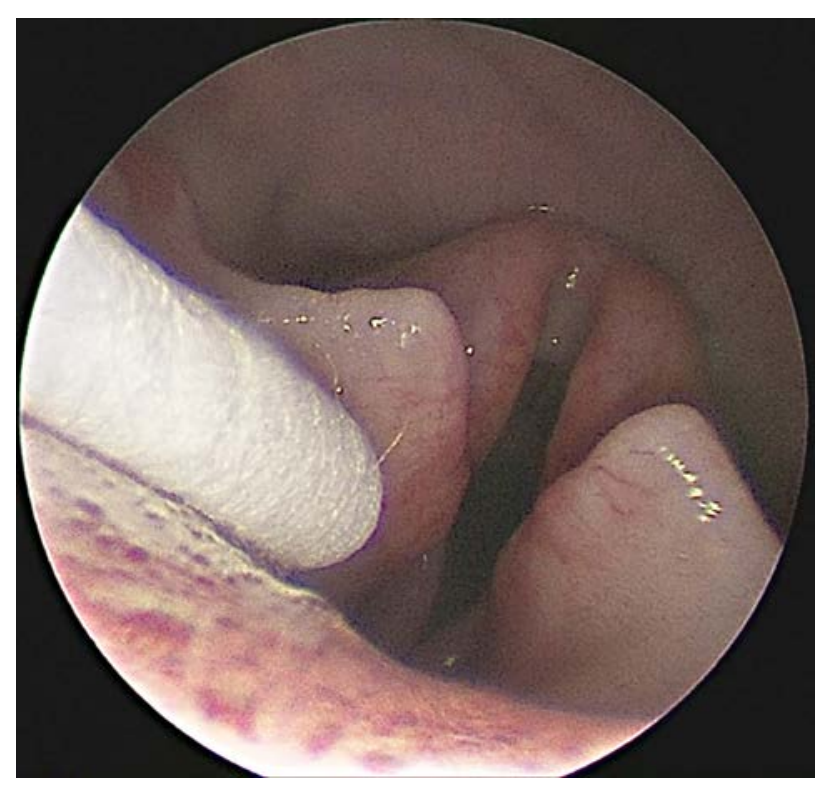

Fig. 2. Swab collecting in the area of larynx 
calisation of foreign bodies [7]. The possibilities of endoscopic surgical interventions of congenital abnormalities, such as: stenotic nostrils, lengthening of the soft palate, stricture of nasopharyngeal compartments and tracheal stenosis which assist in: the therapy management complex, improvement of prognosis and the course and duration of the respiratory disease.

Endoscopic visualisation is the most suitable diagnostic method capable of revealing the sites of pathological changes and the parallel collecting of samples for cytological and microbiological examinations. One of the most frequently used ways of the collection of samples from the suspect regions of the respiratory apparatus involves swabbing under direct endoscopic control [14]. Mucosal biopsy is indicated with the presence of various pathological neoplasms, as well as suspected mycotic diseases. The most effective way of sampling is the nasal sinuses, laryngeal swab (Fig. 2), or bronchoalveolar lavage (BAL). During lavage, the appropriate volume of saline at body temperature will entrap sputum, exudate and surface cells revealing the potential for the development of respiratory infections [2].

The suspicion of the presence of a bacterial infection is frequently formulated during the primary clinical examination. When detecting signs, such as mucopurulent nasal discharge, fever, lethargy, inappetence, the best option according to the International Society for Companion Animal Infectious Diseases (ISCAID) is the empirical administration of doxycycline for 7-10 days as the first line of antibiotic effectiveness against Mycoplasma spp. and Bordetella bronchiseptica. However, much more effective is the therapy of respiratory infections of bacterial origin based on the determination of antibiotic susceptibility of isolated strains. For example, in the presence of secondary bacterial agents such as Pasteurella spp. and Streptococcus spp., the administration of amoxicillin appears to be the most adequate treatment. For the treatment of infection with Staphylococcus spp. it is recommended to use amoxicillin and clavulanic acid since such treatment is not effective against Mycoplasma spp. and Bordetella bronchiseptica [10].

The high prevalence of microbial contamination of the upper airways and increasing resistance to antimicrobials indicate the necessity of the utilisation of the most accurate diagnosis and targeted medicinal therapy. The aim of our clinical study was by means of the targeted endoscopic visualisation of pathological changes, to point out the increased effectiveness of the collection of samples, reliability of bacterial culture examinations and the development of the targeted therapeutic plan, based on the susceptibility of pathogens to the selected antimicrobial preparations.

\section{MATERIALS AND METHODS}

This study was carried out on patients $(n=26)$ exhibiting long-term clinical signs, such as: coughing, difficult breathing, asphyxia, nasal discharge, hyperaemia and depigmentation in the nostril zone. In 18 patients the disease was localised only in the upper airways, while 8 of them showed signs of a disease spreading into the tracheal zone. All patients were diagnosed and treated at the Small animal clinic of the University of Veterinary Medicine and Pharmacy (UVMP) in Košice. A rigid type of endoscope (Karl Storz, Germany) $2 \mathrm{~mm}$ in diameter and $15 \mathrm{~cm}$ in length was used for the examination of the nasal cavity. The trachea and bronchi were examined with a lavage channel endoscope, $2.7 \mathrm{~mm}$ in diameter and $25 \mathrm{~cm}$ in length.

The group of patients with a disease localised in the nasal cavity included the following breeds: Maltese dogs $(\mathrm{n}=4)$, Komondors $(\mathrm{n}=2)$, Dachshunds $(\mathrm{n}=2)$, mongrels $(\mathrm{n}=2)$, Pitbull $(\mathrm{n}=1)$, Hungarian Vizsla $(\mathrm{n}=1)$, Chihuahua $(\mathrm{n}=1)$, and German short-haired pointer $(n=1)$. The mean age of the patients was 6 years. After the targeted endoscopic visualisation of the pathological changes sampled from the nasal cavities of the dogs, they were obtained by swabbing of the nasal mucosa in 14 cases (74 \%): Maltese dogs $(n=4)$, Dachshunds $(n=2)$, Komondors $(n=2)$, mongrels $(n=2)$, Yorkshire terriers $(n=2)$, Pitbull $(n=1)$, and Hungarian Vizsla $(n=1)$. Nasopharyngeal swabs were obtained from 4 dogs (21\%): Yorkshire terriers $(n=2)$, German short-haired pointer $(n=1)$, and Chihuahua $(n=1)$. The sample obtained from a 9 years old Yorkshire terrier male consisted of a swab and the lavage of sinuses with saline (in 1 case $-5 \%$ ).

The group of patients with clinical signs involving the trachea consisted of: Yorkshire terriers $(n=4)$, Dachshunds $(n=2)$, Maltese dog $(n=1)$, and mongrel $(n=1)$. Their mean age was 7 years. Samples from these dogs were obtained in the same way, namely by swabbing the tracheal mucosa.

All patients were subjected to a basic clinical examination which included: sampling of venous blood; evaluation of a roentgenogram of the thoracic cavity in lateral projec- 
tion; and auscultation. Before each targeted endoscopic examination, the animals were sedated utilizing a combination of three anaesthetics administered to the patients intravenously, i. e. (Cepetor $1 \mathrm{mg} \cdot \mathrm{ml}^{-1}$ inj. solution, CP-Pharma, Germany at a dose of $0.015 \mathrm{mg} \cdot \mathrm{kg}^{-1}$ body weight), diazepam (Apaurin $5 \mathrm{mg} \cdot \mathrm{ml}^{-1}$ inj. solution, KRKA, Slovenia at a dose of $0.3 \mathrm{mg} \cdot \mathrm{kg}^{-1}$ b. w.), and propofol (Propofol 10 $\mathrm{mg} \cdot \mathrm{ml}^{-1}$ inj. suspension, Fresenius Kabi, Germany at a dose of $3 \mathrm{mg} \cdot \mathrm{kg}^{-1} \mathrm{~b}$. w.).

For the primary bacterial culture of the samples and isolation of microorganisms we used a non-selective culture medium [Columbia agar (HiMedia, India) enhanced with $5 \%$ sheep blood]. In parallel, as another non-selective medium suitable also for propagation of pigment forming bacteria (such as Pseudomonas aeruginosa and Staphylococcus aureus), we used meat-peptone agar (Nutrient agar No. 2, HiMedia, India). On the basis of the character of growth and haemolytic activity of the isolated bacteria, the colonies were inoculated to the specific selective Endo agar (HiMedia, India) with the focus on enterobacteria, including E. coli, Klebsiella and Proteus, and on to a diagnostic medium Baird-Parker agar (HiMedia, India) for identification of S. aureus. The basic diagnosis included microscopic examination of the native preparations and Gram-stained preparations.

Commercial biochemical tests were used for additional, more accurate identifications. The basic tests used in our study included catalase (3\% hydrogen peroxide) and oxidase test (OxiTest, Erba Lachema, Czech Republic). For the species specification we used EnteroTest 24, NefermTest 24, StaphyTest 24, and Streptotest 24 (Erba Lachema, Czech Republic). The results of the tests were evaluated by means of software TNW ProAuto 7.0.

The identified bacterial strains were subsequently subjected to tests of susceptibility/resistance to antibiotics by means of a modern automatized instrument VITEK ${ }^{\oplus} 2$ intended for the determination of the minimum inhibition concentrations of antibiotics. The results of the tests were interpreted in agreement with the criteria determined by the European Committee on Antimicrobial Susceptibility Testing (EUCAST). The complex microbiological diagnosis was carried out in specialised clinical microbiology laboratories.

\section{RESULTS}

The importance of endoscopic visualisation of the respiratory tract for the diagnosis of acute and chronic diseases was confirmed. This examination revealed a broad range of pathological changes in the majority of the patients. These pathologic manifestations ranged from mucous clumps adhered to the mucous membrane of the respiratory airways, through typical macroscopic hyperaemic manifestations of inflammation, up to anatomical changes observed most frequently in the conchae, nasopharynx and trachea.

The results of the microbiological examinations of the samples collected in this study revealed the prevalence of bacterial strains involved in diseases of the upper respiratory tract. The pathogens found in the highest abundance in the nasal cavity and nasopharynx area were: Enterococcus faecalis (15.6\%), Staphylococcus aureus (12.5\%), Staphylococcus pseudintermedius (9.4\%), Escherichia coli (9.4\%), Bacillus spp. (6.3\%), Pseudomonas aeruginosa (6.3\%) and others (Table 1). In the samples from the trachea, Escherichia coli (20\%), Enterobacter ludwigii (13\%), haemolyticEscherichia coli (13\%) and others (Table 2) were found in the highest abundance.

In addition to the microbiological cultivation of the samples, antibiograms were prepared illustrating the effectiveness of various groups of antibiotics used in veterinary practice. The determination of antibiotic resistance of individual pathogens provided the necessary information for the initiation of targeted individual antibiotic therapy. The results of the antibiotic resistance of contaminants of the nasal cavity and trachea isolated in our study may be seen in Figures 3 and 4 . The effectiveness of individual groups of antibiotics used in the treatment of common infections of the upper respiratory tract in descending sequence were as follows: fluoroquinolones (90.0\%), aminoglycosides (89.5\%), tetracyclines $(83.0 \%)$, trimetoprim-sulphate (81.5\%), phenicols $(77.0 \%)$, cephalosporins $(72.5 \%)$, polymyxin B (63.0\%), penicillins (53.0\%), macrolides (36.0\%), fusidic acid (20.0\%), and lincosamides (11.8\%), see Figure 5. 
Table 1. Bacterial species found in the nasal cavity

\begin{tabular}{|c|c|c|}
\hline Bacterial species & Number of findings & $\%$ \\
\hline Enterococcus faecalis & 5 & 15.6 \\
\hline Staphylococcus aureus & 4 & 12.5 \\
\hline Escherichia coli & 3 & 9.4 \\
\hline Staphylococcus pseudintermedius & 3 & 9.4 \\
\hline Bacillus spp. & 2 & 6.3 \\
\hline Pseudomonas aeruginosa & 2 & 6.3 \\
\hline Acinetobacter baumanii & 1 & 3.1 \\
\hline Acinetobacter pittii & 1 & 3.1 \\
\hline Acinobacter spp. & 1 & 3.1 \\
\hline Aeromonas caviae & 1 & 3.1 \\
\hline Klebsiella pneumoniae & 1 & 3.1 \\
\hline Neisseria zoodegmatis & 1 & 3.1 \\
\hline Pantoea agglomerans & 1 & 3.1 \\
\hline Pasteurella canis & 1 & 3.1 \\
\hline Pasteurella dagmatis & 1 & 3.1 \\
\hline Pasteurella stomatis & 1 & 3.1 \\
\hline Staphylococcus epidermidis & 1 & 3.1 \\
\hline Staphylococcus intermedius & 1 & 3.1 \\
\hline Staphylococcus epidermidis & 1 & 3.1 \\
\hline
\end{tabular}

Table 2. Bacterial species found in the trachea

\begin{tabular}{lcc}
\hline \multicolumn{1}{c}{ Bacterial species } & Number of findings & $\%$ \\
\hline Escherichia coli & 3 & 20.0 \\
Enterobacter ludwigii & 2 & 13.3 \\
Haemolytical E. coli & 2 & 13.3 \\
Enterobacter cloacae & 1 & 6.7 \\
Enterobacter spp. & 1 & 6.7 \\
Beta haemolytic streptococci & 1 & 6.7 \\
Klebsiella pneumoniae & 1 & 6.7 \\
Pasteurella multocida & 1 & 6.7 \\
Pasteurella stomatis & 1 & 6.7 \\
Proteus mirabilis & 1 & 6.7 \\
\hline
\end{tabular}

Table 3. The effect of antimicrobial drugs used in the UVMP hospital on bacteria isolated in the study

\begin{tabular}{|c|c|c|c|c|c|c|c|}
\hline Active ingredient & $\begin{array}{c}\text { Acinetobacter } \\
\text { spp. } \\
(n=3)\end{array}$ & $\begin{array}{c}\text { Pasteurella } \\
\text { spp. } \\
(n=5)\end{array}$ & $\begin{array}{c}\text { Staphylococci } \\
\text { spp. } \\
(n=9)\end{array}$ & $\begin{array}{c}\text { Streptococci } \\
\text { spp. } \\
(n=2)\end{array}$ & $\begin{array}{c}\text { Bacillus } \\
\text { spp. } \\
(\mathrm{n}=2)\end{array}$ & $\begin{array}{l}\text { Enterobacteriacae } \\
\qquad(n=4)\end{array}$ & $\begin{array}{l}\text { E. coli } \\
(\mathrm{n}=8)\end{array}$ \\
\hline Benzyl penicillin & - & $0 \%$ & $28 \%$ & $0 \%$ & - & $100 \%$ & $100 \%$ \\
\hline Cephalexin & $100 \%$ & $0 \%$ & $14 \%$ & $0 \%$ & - & $100 \%$ & $29 \%$ \\
\hline Enrofloxacin & $0 \%$ & $0 \%$ & $13 \%$ & $0 \%$ & - & $0 \%$ & $25 \%$ \\
\hline $\begin{array}{l}\text { Amoxicillin } \\
\text { clavulanic acid }\end{array}$ & $100 \%$ & $0 \%$ & $25 \%$ & $0 \%$ & - & $100 \%$ & $25 \%$ \\
\hline $\begin{array}{l}\text { Amoxicillin } \\
\text { clavulanic acid }\end{array}$ & $100 \%$ & $0 \%$ & $25 \%$ & $0 \%$ & - & $100 \%$ & $25 \%$ \\
\hline Gentamycin & $0 \%$ & $0 \%$ & $0 \%$ & $100 \%$ & - & $0 \%$ & $13 \%$ \\
\hline
\end{tabular}




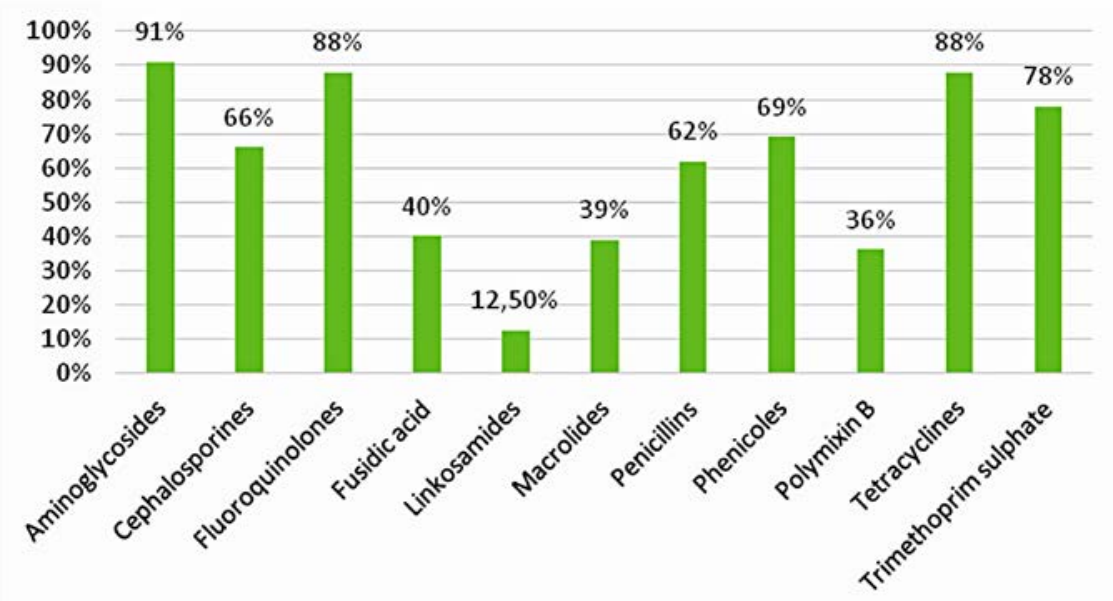

Fig. 3. The effect of antibiotics against bacterial species found in the nasal cavity

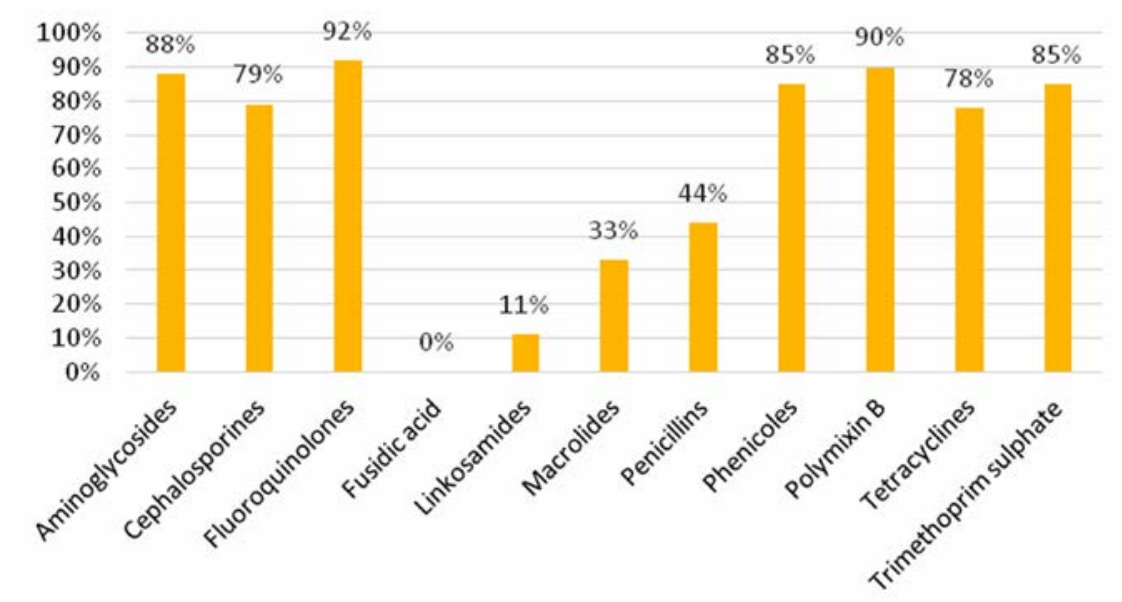

Fig. 4. The effect of antibiotics against bacterial species found in the trachea

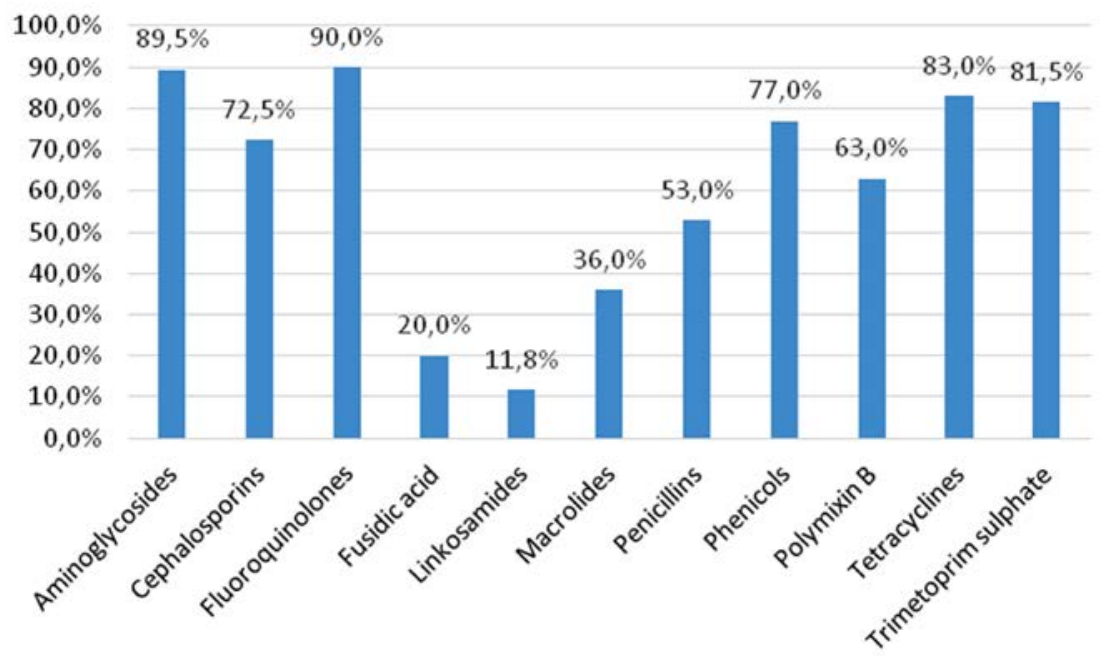

Fig. 5. The effectiveness of antibiotics used in the treatment of common infections of the upper respiratory tract 


\section{DISCUSSION}

Numerous infectious agents are responsible for causing primary or secondary respiratory diseases in dogs. Clinical signs may vary from mild dyspnoea, sneezing, and coughing to severe pneumonia with systemic manifestations. Depending on the etiological agent, the gross and microscopic changes observed during these infections can be rather unspecific or have highly characteristic patterns [15].

There are many diagnostic tools available on the market that can be used for the identification of respiratory agents. The development of molecular diagnostic tools allows the rapid identification of a wide variety of pathogens and the establishment of more accurate treatments [16]. Most common bacterial cultures from the nasal cavity can be performed from specimens obtained by nasal swabbing. However nasal lavage specimens gave a higher sensitivity for bacterial growth than tissue biopsy specimens and swabs [8]. In comparison with dogs, bacterial culture results can be difficult or impossible to interpret as bacteria can also be cultured from the nasal cavity of healthy cats. However multidrug-resistant bacteria can colonize and be grown from the nasal passages in the absence of infection [10]. Bacterial cultures from the respiratory tract can be performed from specimens obtained from oropharyngeal swabs, tracheal wash, or bronchoalveolar lavage (BAL). Collection of a tracheal wash or bronchoalveolar lavage specimen is indicated in dogs with more severe clinical signs or evidence of pneumonia [13]. Some clinicians recommend quantitative or semiquantitative bacterial cultures of BAL fluid, and it has been reported that counts greater than $10^{4}$ colony forming units (CFU.ml ${ }^{-1}$ ) (or grown from primary culture) represent true infection whereas less than 103 CFU.ml ${ }^{-1}$ (or grown from subculture) represent contamination [12].

The treatment of upper respiratory diseases is symptomatic; however, due to the common occurrence of secondary infections with a broad spectrum of bacteria, antibiotic treatment is the first therapeutic approach. Antimicrobial agents should be selected based on culture and sensitivity tests of airway specimens collected by bronchoscopy [14]. Endoscopy is a valuable diagnostic approach to the upper and lower respiratory tract, because it allows direct visualisation and sample collection. However, each anatomical region may require a range of specialized technical equipment and varying levels of experience to access and eval- uate specific sites. Each procedure and normal appearance like cytology and culture results from each region will enhance the diagnostic success [5].

By means of endoscopic diagnosis and direct collection of samples from the nasal cavity $(n=18)$, nasopharynx $(\mathrm{n}=4)$ and trachea $(\mathrm{n}=8)$ of 26 animal patients, microbial cultures allowed us to identify 27 bacterial species in a total number of 47 bacterial findings. The most frequently isolated Gram-negative bacteria were: Escherichia coli (17.0\%), Pasteurella spp. (10.6\%), members of the family-Enterobacteriacae (10.6\%), Acinetobacter spp. (6.4\%), Klebsiella pneumoniae (4.2\%), Pseudomonas aeruginosa (4.2\%), Proteus mirabilis (2.1\%), Aeromonas caviae (2.1\%), and Neisseria zoodegmatis (2.1\%). Of the Gram-positive bacteria, we detected most frequently: Staphylococcus aureus (8.5\%), Staphylococcus pseudintermedius (6.4\%), Streptococcus spp. (6.4\%), Staphylococcus spp. (4.2\%) and Bacillus spp. (4.2\%). Our results resemble those published by $\mathrm{A} \mathrm{d}$ a s zek et al. [1], showing relatively frequent involvement of the pathogenic bacteria E. coli, Klebsiella spp. and Staphylococcus spp. in the respiratory tract infections in the dogs.

In the relevant study conducted by $\mathrm{D}$ a o d u et al. [6], the authors isolated 222 bacterial species. The most frequently identified species were: Staphylococcus spp. (21.7\%), E. coli (18.5\%), Proteus spp. (17.1\%), Acinetobacter spp. (9.0\%), Pseudomonas spp. (6.8\%) and Streptococcus spp. (5.4\%). The results of culture studies of samples from 90 nasal swabs published by A y o d hy a et al. [2] revealed various types of bacteria in mild cases: E. coli (63.33\%), Klebsiella spp. (30\%) and mixed infections (E. coli and Klebsiella spp.) (6.67\%), in 19, 9 and 2 dogs (out of 30 samples), respectively. Similarly, in the case of moderate respiratory diseases, samples revealed the presence of various bacteria: E. coli (30\%), Klebsiella spp. (33.33\%), Streptococcus spp. (30\%) and mixed infections (Klebsiella spp. and Streptococcus spp.) (6.67\%), in 9, 10, 9 and 2 dogs (out of 30 samples), respectively. In the case of severe respiratory diseases, various bacteria were identified: E. coli (6.67 \%), Klebsiella spp. (3.33\%), Streptococcus spp. (46.67 \%), Staphylococci spp. (40\%) and mixed infection (E. coli and Streptococcus spp.) (3.33\%) in 2, 1, 14, 12 dogs and $1 \mathrm{dog}$ (out of 30 samples), respectively. The study conducted by $\mathrm{C} \mathrm{h}$ a r k r a b a r th i [4] showed that Klebsiella pneumoniae and E. coli spp. were the common bacteria involved in respiratory infectious diseases. 
The information related to microbial contamination of the upper respiratory tract obtained in our study were subsequently used for the determination of antibiotic resistance of the isolates against 11 groups of antibiotics. The effectiveness in descending sequence was as follows: aminoglycosides (89.8\%), fluoroquinolones (89.5\%), tetracyclines $(84.2 \%)$, trimetoprim-sulphate $(80.7 \%)$, phenicols $(77,0 \%)$, cephalosporins $(71.2 \%)$, polymyxin B $(67,8 \%)$, penicillins (55,0\%), macrolides (35.6\%), fusidic acid (16.6\%) and lincosamides (11.6\%). In the study published by $\mathrm{D}$ a dou et al. [6] the effectiveness of fluoroquinolones on Gram-positive and Gram-negative bacteria ranged from $78.4 \%$ to $93.3 \%$. The effectiveness of cephalosporines against Gram-positive bacteria reached $31.7 \%$ while the effect of penicillins (e.g. amoxicillin), as the first line antibiotics, was paradoxically rather low. It reached $15.0 \%$ against Gram-positive and $37.7 \%$ against Gram-negative strains.

\section{CONCLUSIONS}

Chronic and long-term diseases of the upper respiratory tract require correct and targeted diagnosis. Direct visualisation of the respiratory airways is capable of ensuring precise sampling, accurate diagnosis and the development of the subsequent rational therapeutic plan. Direct targeted collection of samples is advantageous with respect to the elimination of the complex multifactorial influences and contribution to the therapy effectiveness. Microbiological culture methods focused on pathogenic bacteria constitute an inseparable part of the diagnosis of respiratory diseases especially with respect to prevention of increasing antibiotic resistance and incidence of chronic conditions. Although the financial demands of endoscopic diagnostic approach are moderately increased, it has been confirmed that the results obtained in this way are reliable. In practice it may result in reduced administration of therapeutically effective medications, more effective treatment and prevention of an increase in antibiotic resistance and thus, also reduced load on the patient.

\section{REFERENCES}

1. Adaszek, L., Gorna, M., Zietek, J., Kutrzuba, J., Winiarczyk, S., 2009: Bacterial nosocomial infections in dogs and cats. Zycie Weterynaryjne, 84, 805-808.

2. Ayodhya, S., Rao, D. T., Reddy, Y. N., Sundar, N. S., Kumar, V. G., 2013: Isolation and characterization of bacteria from canine respiratory diseases in and around Hyderabad city, Andhra Pradesh, India. Vet. World, 6, 9, 601-604. DOI: 10. 5455/vetworld.2013.601-604.

3. Carey, S. A., 2019: Canine Infectious Respiratory Disease Complex: Host, Pathogen, and Environmental Interactions. Retrieved from https://www.michvma.org/resources/Documents/MVC/2018\%20Proceedings/carey_05.pdf.

4. Charkrabarthi, A., 2009: Textbook of Clinical Veterinary Medicine. 3rd edn., Ludhiana, Kalayani Publishers, Punjub, India, 327-372.

5. Creevy, K. E., 2009: Airway evaluation and flexible endoscopic procedures in dogs and cats: laryngoscopy, transtracheal wash, tracheobronchoscopy, and bronchoalveolar lavage. Vet. Clin. North Am. Small Anim. Pract., 39, 5, 869-880. DOI: 10.1016/j.cvsm.2009.05.001.

6. Daodu, O. B., Amosun, E. A., Oluwayelu, D. O., 2017: Antibiotic resistance profiling and microbiota of the upper respiratory tract of apparently healthy dogs in Ibadan, South west Nigeria. Afr. J. Infect. Dis., 11, 1, 1-11. DOI: 10.4314/ ajid.v11i1.4533.

7. Elie, M., Sabo, M., 2006: Basics in canine and feline rhinoscopy. Clin. Tech. Small Anim. Pract., 21, 2, 73-77. DOI: 10. 1053/j.ctsap.2005.12.011.

8. Johnson, L. R., Kass, P. H., 2009: Effect of sample collection methodology on nasal culture results in cats. J. Feline Med. Surg., 11, 645-649. DOI: 10.1016/j.jfms.2008.12.004.

9. Kuehn, N. F., 2013: Tracheobronchitis in Small Animals. Retrieved in Sept. 2020, from https://www.msdvetmanual.com/ respiratory-system/respiratory-diseases-of-small-animals/ tracheobronchitis -in-small-animals.

10. Lappin, M. R., Blondeau, J., Boothe, D., Breitschwerdt, E. B., Guardabassi, L., Lloyd, D. H., et al., 2017: Antimicrobial use guidelines for treatment of respiratory tract disease in dogs and cats: Antimicrobial Guidelines Working Group of the International Society for Companion Animal Infectious Diseases. J. Vet. Intern. Med., 31, 2, 279-294. DOI: 10.1111/ jvim.14627. 
11. Levkut, M., Švický, E., Lenhardt, L., Ševčíková, Z., Revajová, V., Herich, R., 2007: Special Veterinary Pathology (In Slovak). Vol. I. University of Veterinary Medicine and Pharmacy in Košice, Slovakia, 239 pp.

12. Padrid P., 2000: Pulmonary diagnostics. Vet. Clin. North Am. Small Anim. Pract., 30, 6, 1187-1206. DOI: 10.1016/s01955616(00)06008-3.

13. Reagan, K. L., Sykes, J. E., 2020: Canine infectious respiratory disease. Vet. Clin. North Am. Small Anim. Pract., 50, 2, 405-418. DOI: 10.1016/j.cvsm.2019.10.009.

14. Sumner, C. M., Rozanski, E. A., Sharp, C. R., Shaw, S. P., 2011: The use of deep oral swabs as a surrogate for transoral tracheal wash to obtain bacterial cultures in dogs with pneumonia. J. Vet. Emerg. Crit. Care, 21, 5, 515-520. DOI: 10. 1111/j.1476-4431.2011. 00670.x.
15. Vieson, M. D., Pineyro, P., LeRoith, T., 2012: A review of the pathology and treatment of canine respiratory infections. Vet. Med., 3, 25-39. DOI: 10.2147/VMRR.S25021.

16. Windsor, R. C., Johnson, L. R., Sykes, J. E., Drazenovich, T. L., Leutenegger, C. M., De Cock, H. E. V., 2006: Molecular detection of microbes in nasal tissue of dogs with idiopathic lymphoplasmacyticrhinitis.J. Vet.Intern.Med.,20,2,250-256. DOI: 10.1892/0891-6640(2006)20[250:MDOMIN]2.0.CO;2

Received January 4, 2020

Accepted February 22, 2021 